\title{
Particle production at very low and intermediate transverse momenta in d+Au and Au+Au collisions
}

\author{
Adam Trzupek ${ }^{3}$ for the PHOBOS Collaboration
}

B.Alver ${ }^{4}$, B.B.Back ${ }^{1}$, M.D.Baker ${ }^{2}$, M.Ballintijn ${ }^{4}$, D.S.Barton ${ }^{2}$, R.R.Betts ${ }^{6}$, A.A.Bickley ${ }^{7}$, R.Bindel ${ }^{7}$, A.Budzanowski ${ }^{3}$, W.Busza ${ }^{4}$, A.Carroll ${ }^{2}$, Z.Chai ${ }^{2}$, V.Chetluru ${ }^{6}$, M.P.Decowski ${ }^{4}$, E.García $^{6}$, T.Gburek ${ }^{3}$, N.George ${ }^{2}$, K.Gulbrandsen ${ }^{4}$, S.Gushue ${ }^{2}$, C.Halliwell ${ }^{6}$, J.Hamblen ${ }^{8}$, G.A.Heintzelman ${ }^{2}$, C.Henderson ${ }^{4}$, I.Harnarine ${ }^{6}$, D.J.Hofman ${ }^{6}$, R.S.Hollis ${ }^{6}$, R.Hołyński ${ }^{3}$, B.Holzman ${ }^{2}$, A.Iordanova ${ }^{6}$, E.Johnson ${ }^{8}$, J.L.Kane ${ }^{4}$, N.Khan ${ }^{8}$, W.Kucewicz ${ }^{6}$, P.Kulinich ${ }^{4}$, C.M.Kuo ${ }^{5}$, W.Li ${ }^{4}$, W.T.Lin ${ }^{5}$, C.Loizides ${ }^{4}$, S.Manly ${ }^{8}$, A.C.Mignerey ${ }^{7}$, R.Nouicer ${ }^{2,6}$,

A.Olszewski ${ }^{3}$, R.Pak $^{2}$, I.C.Park ${ }^{8}$, C.Reed ${ }^{4}$, L.P.Remsberg ${ }^{2}$, M.Reuter $^{6}$, E.Richardson ${ }^{7}$, C.Roland ${ }^{4}$, G.Roland ${ }^{4}$, L.Rosenberg ${ }^{4}$, J.Sagerer $^{6}$, P.Sarin ${ }^{4}$, P.Sawicki ${ }^{3}$, I.Sedykh ${ }^{2}$, W.Skulski ${ }^{8}$, C.E.Smith ${ }^{6}$, M.A.Stankiewicz ${ }^{2}$, P.Steinberg ${ }^{2}$, G.S.F.Stephans ${ }^{4}$, A.Sukhanov ${ }^{2}$, A.Szostak ${ }^{2}$, J.-L.Tang ${ }^{5}$, M.B.Tonjes ${ }^{7}$, A.Trzupek ${ }^{3}$, C.Vale ${ }^{4}$, G.J.van Nieuwenhuizen ${ }^{4}$, S.S. Vaurynovich ${ }^{4}$, R.Verdier ${ }^{4}$, G.I.Veres ${ }^{4}$, P.Walters ${ }^{8}$, E.Wenger ${ }^{4}$, D.Willhelm ${ }^{2}$, F.L.H.Wolfs ${ }^{8}$, B.Wosiek ${ }^{3}$, K.Woźniak ${ }^{3}$, A.H.Wuosmaa ${ }^{1}$, S.Wyngaardt ${ }^{2}$, B.Wysłouch ${ }^{4}$

${ }^{1}$ Argonne National Laboratory, Argonne, IL 60439-4843, USA

${ }^{2}$ Brookhaven National Laboratory, Upton, NY 11973-5000, USA

${ }^{3}$ Institute of Nuclear Physics PAN, Kraków, Poland

${ }^{4}$ Massachusetts Institute of Technology, Cambridge, MA 02139-4307, USA

${ }^{5}$ National Central University, Chung-Li, Taiwan

${ }^{6}$ University of Illinois at Chicago, Chicago, IL 60607-7059, USA

${ }^{7}$ University of Maryland, College Park, MD 20742, USA

${ }^{8}$ University of Rochester, Rochester, NY 14627, USA

The transverse momentum $\left(p_{T}\right)$ spectra of identified charged particles have been measured at very low and intermediate transverse momenta in $\mathrm{Au}+\mathrm{Au}$ collisions at $\sqrt{s_{N N}}=62.4 \mathrm{GeV}$ and $\mathrm{d}+\mathrm{Au}$ collisions at $\sqrt{s_{N N}}=200 \mathrm{GeV}$ using the PHOBOS detector at RHIC. New results on charged particle production at very low $p_{T}$ in central $\mathrm{Au}+\mathrm{Au}$ collisions at $\sqrt{s_{N N}}=200 \mathrm{GeV}$ in the centrality intervals $0-6 \%$ and $6-15 \%$ are presented. A comparison of the PHOBOS low- $p_{T}$ data with predictions of a recent optical model is shown. The shapes of $m_{T}$ spectra for $\mathrm{d}+\mathrm{Au}$ and $\mathrm{Au}+\mathrm{Au}$ collisions are compared.

\section{Introduction}

In nucleus-nucleus collisions, an enhanced production of low- $p_{T}$ particles could signal new long-wavelength physics phenomena [ 1, 2]. It is also expected that yields of particles with higher masses, like protons and antiprotons can be modified due to collective transverse expansion of the system [ 3, 4]. Measurements at very low $p_{T}$ can also provide a critical test for models predicting a pronounced modification of the low- $p_{T}$ particle emission pattern, e.g. [5]. 
The PHOBOS experiment has the unique capability to measure charged particles at transverse momenta as low as 30, 90 and $140 \mathrm{MeV} / \mathrm{c}$ for charged pions, kaons and for protons and antiprotons, respectively, using a multi-layer, magnetic spectrometer. Yields at very low transverse momenta are determined using a reconstruction procedure developed to look for particles which range out in the fifth silicon layer of the PHOBOS spectrometer. A description of the "stopping algorithm" is presented in [6]. At intermediate $p_{T}$, particle momentum and charge are obtained from the curvature of particle trajectories in a 2T magnetic field and particle identification is provided by the specific energy loss $(d E / d x)$ in the spectrometer and by Timeof-Flight detectors. Details on tracking, particle identification, event selection and centrality determination in the PHOBOS detector can be found in [7].

\section{2. $\mathrm{p}_{\mathrm{T}}$ spectra in $\mathrm{Au}+\mathrm{Au}$ collisions at $\sqrt{\mathrm{s}_{\mathrm{NN}}}=62.4 \mathrm{GeV}$}

The preliminary particle yields for $\pi^{ \pm}$, $K^{ \pm}, p$ and $\bar{p}$ are presented in Fig. 1 for three centrality intervals: $0-15 \%, 15-30 \%$ and $30-50 \%$. The data are corrected for detector effects (acceptance, efficiency, momentum resolution) and background particles including feed-down from weak decays and secondary particles produced in the beam pipe and detector material. The rapidity coverage of measured yields extends from about 0.4 to 1.4 for $\pi^{ \pm}$, from 0.2 to 1.2 for $K^{ \pm}$and from 0.2 to 1.1 for $p$ and $\bar{p}$. The preliminary results on antiparticle to particle ratios have been obtained for the $15 \%$ most central collisions. The results of $0.84 \pm 0.02$ (stat.) \pm 0.08 (syst.) for $K^{-} / K^{+}$and $0.37 \pm 0.01$ (stat.) \pm 0.06 (syst.) for $\bar{p} / p$ fit smoothly into the energy evolution of antiparticle to particle ratios from the AGS up to the highest RHIC energy.

Low- $p_{T}$ yields of $\left(\pi^{+}+\pi^{-}\right),\left(K^{+}+K^{-}\right)$ and $(p+\bar{p})$ near mid-rapidity in $\mathrm{Au}+\mathrm{Au}$ collisions at $\sqrt{s_{N N}}=62.4 \mathrm{GeV}$, corrected for detector effects and background particles, are shown in Fig. 2 in the same centrality bins. One can see that $\left(K^{+}+K^{-}\right)$ and $(p+\bar{p})$ yields are quite consistent with extrapolations of blast wave functions (BWF) [ 4] fitted to the spectra at higher transverse momenta. Some disagreement between the measured yield of pions and BWF at low $p_{T}$ could be attributed to a contribution from resonances which is not included in the model. A similar behavior was observed for $p_{T}$ yields measured in the $15 \%$ most central $\mathrm{Au}+\mathrm{Au}$ collisions at 
$\sqrt{s_{N N}}=200 \mathrm{GeV}$ [ 6], indicating that at both energies no significant enhancement of particle production is observed at very low $p_{T}$. Also, a flattening of the $(p+\bar{p})$ spectra down to very low transverse momentum is observed. This could be a consequence of collective transverse expansion of the medium created in heavy ion collisions at RHIC.
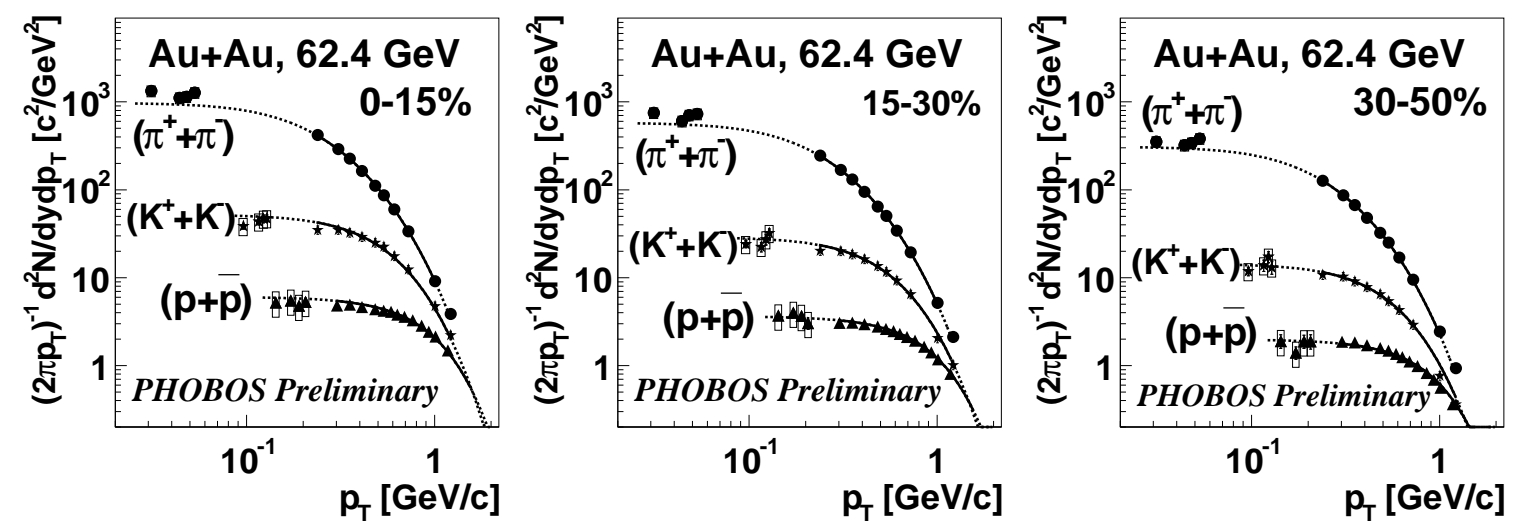

Figure 2. $\left(\pi^{+}+\pi^{-}\right),\left(K^{+}+K^{-}\right)$and $(p+\bar{p})$ yields at very low $p_{T}$ in Au+Au collisions at $\sqrt{s_{N N}}=62.4 \mathrm{GeV}$. Blast wave fits to the intermediate $p_{T}$ data (solid lines) are extrapolated to low $p_{T}$ (dashed lines).

\section{Low- $p_{T}$ yields in central Au+Au collisions at $\sqrt{\mathrm{S}_{\mathrm{NN}}}=200 \mathrm{GeV}$}

The spectra of $\left(\pi^{+}+\pi^{-}\right),\left(K^{+}+K^{-}\right)$and $(p+\bar{p})$ at very low transverse momentum in the $15 \%$ most central $\mathrm{Au}+\mathrm{Au}$ collisions at $\sqrt{s_{N N}}=200 \mathrm{GeV}$, measured in the PHOBOS experiment, are presented in [6]. In order to confront the extrapolations from a recent optical model [5], which were available only for more central collisions, with measurements, the published data sample was split into two finer centrality bins. Fig. [3]shows the $p_{T}$ yields, corrected for detector effects

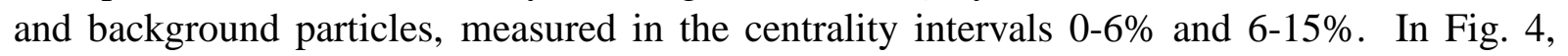
the pion yield measured in the $6 \%$ most central $\mathrm{Au}+\mathrm{Au}$ collisions is compared to the optical model predictions for the spectrum of negative pions at mid-rapidity. The originally published
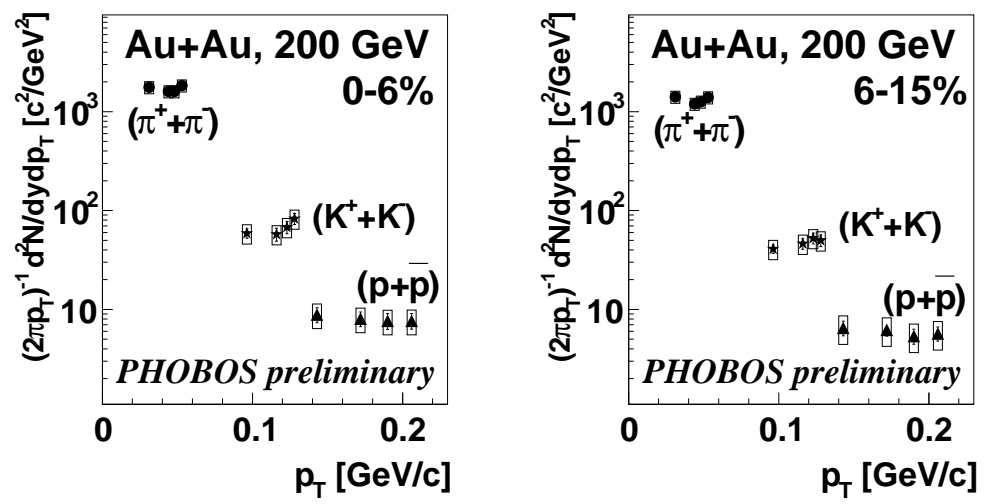

Figure 3. $\left(\pi^{+}+\pi^{-}\right),\left(K^{+}+K^{-}\right)$and $(p+\bar{p})$ yields at very low $p_{T}$ in $0-6 \%$ and $6-15 \%$ central Au+Au collisions at $\sqrt{s_{N N}}=200 \mathrm{GeV}$.

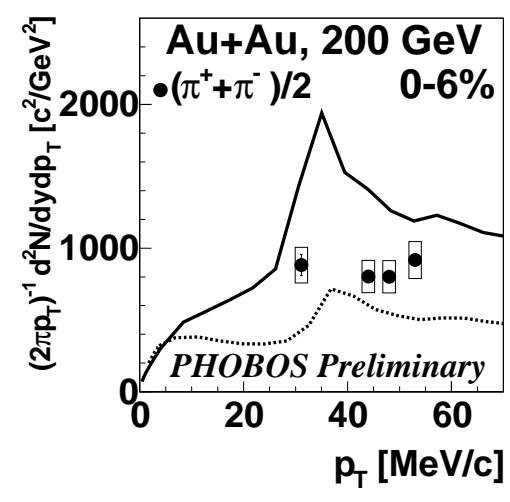

Figure 4. Optical model predictions [5, 8] for pion spectra at low $p_{T}$ compared to PHOBOS data (see text for details). 

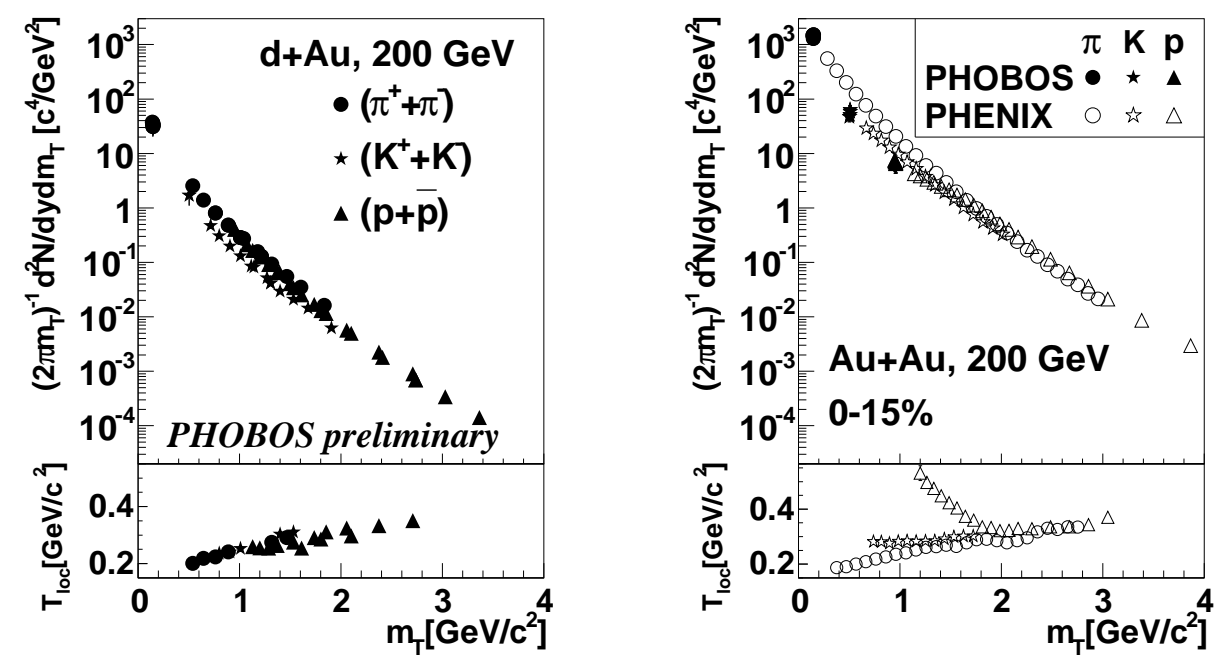

Figure 5. $m_{T}$ spectra of $\left(\pi^{+}+\pi^{-}\right),\left(K^{+}+K^{-}\right)$and $(p+\bar{p})$ at very low and intermediate $p_{T}$ measured in $\mathrm{d}+\mathrm{Au}$ (left plot) and central $\mathrm{Au}+\mathrm{Au}$ collisions (right plot). Inverse local slope parameters of $m_{T}$ spectra are shown in the lower panels of each figure.

extrapolation is shown by the dashed curve, while the solid curve depicts the recently modified model calculations $[5,8]$.

\section{4. $\mathrm{m}_{\mathrm{T}}$ scaling}

It is interesting to compare the particle yields at very low and intermediate $p_{T}$ in $\mathrm{d}+\mathrm{Au}$ and central $\mathrm{Au}+\mathrm{Au}$ collision at the same energy of $\sqrt{s_{N N}}=200 \mathrm{GeV}$. Yields of $\left(\pi^{+}+\pi^{-}\right),\left(K^{+}+\right.$ $\left.K^{-}\right)$and $(p+\bar{p})$ in $\mathrm{d}+\mathrm{Au}$ collisions, corrected for detector effects and background particles, are shown in Fig. 5. One can see that in d+Au collisions $\left(\pi^{+}+\pi^{-}\right)$and $(p+\bar{p}) m_{T}$ spectra are similar while the $\left(K^{+}+K^{-}\right)$spectrum is systematically lower (by a factor of about 2 ) due to strangeness suppression. The $m_{T}$ spectra for the $15 \%$ most central Au+Au collisions measured by the PHOBOS [ 6] and PHENIX [ 9] experiments at very low and intermediate tranverse momenta, respectively, are also shown in Fig. 5 In order to compare the shapes of the $m_{T}$ spectra, inverse local slope parameters were calculated by fitting locally exponential functions to each spectrum (see bottom panels of Fig. 5). We can see that for d+Au collisions local slopes are similar for all particle species both at low and intermediate $p_{T}$. In contrast, $\mathrm{Au}+\mathrm{Au}$ spectral shapes are similar at higher transverse masses $\left(m_{T}>1.7 \mathrm{GeV}\right)$ while at low $m_{T}$ a flattening of $\left(K^{+}+K^{-}\right)$and $(p+\bar{p})$ spectra is observed. This flattening of the $(p+\bar{p})$ spectrum is significantly stronger than the one observed for the spectra of charged kaons. One can also see that the $m_{T}$ dependence of the local slopes of the $\left(\pi^{+}+\pi^{-}\right) m_{T}$ spectrum for Au+Au collision is consistent with that found for the local slopes of $\left(\pi^{+}+\pi^{-}\right),\left(K^{+}+K^{-}\right)$and $(p+\bar{p})$ spectra in $\mathrm{d}+\mathrm{Au}$ collisions.

\section{Summary}

Yields of pions, kaons and protons and antiprotons at very low and intermediate $p_{T}$ near mid-rapidity in $\mathrm{Au}+\mathrm{Au}$ collisions at $\sqrt{s_{N N}}=62.4$ and $200 \mathrm{GeV}$, measured in the PHOBOS 
experiment, indicate that there is no evidence for enhanced production of particles at very low transverse momentum. The pion low- $p_{T}$ data can constrain the recent optical model predictions. A significant flattening of the $(p+\bar{p}) m_{T}$ spectrum down to very low $p_{T}$ is observed in central $\mathrm{Au}+\mathrm{Au}$ collisions at both energies which could be a consequence of the collective transverse expansion of the system. In $\mathrm{d}+\mathrm{Au}$ collisions, no flattening is observed and the shapes of the $m_{T}$ spectra are similar at very low and intermediate $p_{T}$.

This work was partially supported by U.S. DOE grants DE-AC02-98CH10886, DE-FG0293ER40802, DE-FC02-94ER40818, DE-FG02-94ER40865, DE-FG02-99ER41099, and W-31109-ENG-38, by U.S. NSF grants 9603486, 0072204, and 0245011, by Polish KBN grant 1P03B-062-27(2004-2007), by NSC of Taiwan Contract NSC 89-2112-M-008-024, and by Hungarian OTKA grant (F 049823).

\section{REFERENCES}

1. W. Busza, 'Particle Production in Highly Excited Matter", Plenum Press, New York, 1993, p. 149.

2. J. Bjorken, Acta Phys. Pol. B28 (1997) 2773.

3. P.F. Kolb and R. Rapp, Phys. Rev. C67 (2003) 044903.

4. E. Schnedermann, J. Sollfrank and U. Heinz, Phys. Rev. C48 (1993) 2462.

5. J.G. Cramer, G.A.Miller, J.M.S. Wu and J. Yoon, Phys. Rev. Lett. 94 (2005) 102302.

6. B.B. Back, et al., (PHOBOS Collab.), Phys. Rev. C70 (2004) 051901(R).

7. B.B. Back, et al., (PHOBOS Collab.), Nucl. Phys. A757 (2005) 28.

8. B.B. Back, et al., (PHOBOS Collab.), nucl-ex/0506008, J.G. Cramer, 2005, (private communication, erratum to be published).

9. S.S. Adler, et al., (PHENIX Collab.), Phys. Rev. C69 (2004) 034909. 